\title{
The effect of oxidative stress polymorphisms on the association between long-term black carbon exposure and lung function among elderly men
}

\author{
Irina Mordukhovich, ${ }^{1}$ Johanna Lepeule, ${ }^{1,2,3,4}$ Brent A Coull, ${ }^{5}$ David Sparrow, ${ }^{6}$ \\ Pantel Vokonas, ${ }^{6}$ Joel Schwartz ${ }^{1,7}$
}

For numbered affiliations see end of article.

\section{Correspondence to}

Dr Irina Mordukhovich, Exposure, Epidemiology and Risk, Program, Department of Environmental Health, Harvard School of Public Health, Landmark Center, 401 Park Dr, Boston MA 02215, USA: imordukh@hsph.harvard.edu

Received 25 August 2014 Revised 31 October 2014 Accepted 4 November 2014 Published Online First 20 November 2014

\section{CrossMark}

To cite: Mordukhovich 1 Lepeule J, Coull BA, et al. Thorax 2015;70:133-137.
ABSTRACT

Background Black carbon (BC) is a pro-oxidant, traffic-related pollutant linked with lung function decline. We evaluated the influence of genetic variation in the oxidative stress pathway on the association between long-term BC exposure and lung function decline. Methods Lung function parameters (FVC and $\mathrm{FEV}_{1}$ ) were measured during one or more study visits between 1995 and 2011 ( $n=651$ participants) among an elderly cohort: the Normative Aging Study. Residential BC exposure levels were estimated using a spatiotemporal land use regression model. We evaluated whether oxidative stress variants, combined into a genetic score, modify the association between 1-year and 5-year moving averages of $B C$ exposure and lung function levels and rates of decline, using linear mixed models.

Results We report stronger associations between longterm $B C$ exposure and increased rate of lung function decline, but not baseline lung function level, among participants with higher oxidative stress allelic risk profiles compared with participants with lower risk profiles. Associations were strongest when evaluating 5-year moving averages of BC exposure. A $0.5 \mu \mathrm{g} / \mathrm{m}^{3}$ increase in 5 -year $\mathrm{BC}$ exposure was associated with a $0.1 \%$ yearly increase in $\mathrm{FVC}(95 \% \mathrm{Cl}-0.5$ to 0.7$)$ among participants with low genetic risk scores and a $1.3 \%$ yearly decrease $(95 \% \mathrm{Cl}-1.8$ to -0.8$)$ among those with high scores ( $p$-interaction $=0.0003$ ).

Discussion Our results suggest that elderly men with high oxidative stress genetic scores may be more susceptible to the effects of $B C$ on lung function decline. The results, if confirmed, should inform air-quality recommendations in light of a potentially susceptible subgroup.

\section{INTRODUCTION}

Air pollution exposure has been linked to decreased lung function in epidemiological and laboratory studies, particularly when evaluating particulate pollutants. ${ }^{1-4}$ Impaired lung function, in turn, yields reduced quality of life and increased mortality risk. ${ }^{5}$ The association between air pollution and lung function among the elderly, who are particularly susceptible to the health effects of particles, has been sparsely studied. Recent results from the Normative Aging Study (NAS), a population of elderly men, linked long-term exposure to black carbon (BC) with the rate of lung function decline. $^{2} \quad \mathrm{BC}$ is an incomplete combustion

\section{Key messages}

What is the key question?

- Is the association between exposure to ambient black carbon, a traffic-related pollutant and lung function decline modified by genetic variants in the oxidative stress pathway?

\section{What is the bottom line?}

- We report stronger associations between black carbon exposure and rate of lung function decline among participants with a higher oxidative stress allelic risk profile.

\section{Why read on?}

- This is the first study to evaluate whether oxidative stress-related genetic variants modify the association between black carbon exposure and lung function, and the results, if confirmed, may be suggestive of a vulnerable subpopulation: elderly men with higher oxidative stress allelic risk profiles.

by-product considered to be a proxy for all traffic-related particles. ${ }^{6}$

Oxidative stress is associated with lung function decline. ${ }^{7}$ Traffic particles, including BC, induce oxidative stress systemically and in the lung. ${ }^{8}{ }^{9}$ Hence, oxidative stress is a likely mechanism underlying the association between traffic particles and lung function. While it is of interest to evaluate whether functional genetic variants within this pathway could modify the BC-lung function association, no study has performed such an analysis. A novel genetic score for oxidative stress-related genetic variants was previously calculated in the NAS to evaluate the role of biological mechanisms while reducing the number of statistical comparisons. ${ }^{10}$

We hypothesised that, in that same cohort of elderly men, the NAS, associations between BC and lung function decline would be stronger among participants with higher oxidative stress allelic risk profiles. To investigate this hypothesis, we evaluated whether previously reported associations between long-term (1-year and 5-year) moving averages of residential $\mathrm{BC}$ exposure and lung function levels and rates of decline were modified by participants' oxidative stress genetic scores. 


\section{MATERIALS AND METHODS Study population}

Our study included 651 men, who underwent 1933 study visits between October 1995 and August 2011. These men were enrolled in the Veterans Administration NAS, a prospective cohort study described in detail previously. ${ }^{11}$ Briefly, this closed cohort was established in 1963 and enrolled 2280 adult male volunteers, free of chronic medical conditions, who were living in the Greater Boston area. Of the 2280 participants enrolled in 1963, many have been lost to follow-up, primarily due to death or moving out of the study area. There were 1062 active NAS study participants during the time period of interest. Of these, 653 participants had complete information regarding long-term residential BC exposures, lung function measurements and oxidative stress genetic profiles for one or more study visits. Two of these 653 participants were missing information regarding educational attainment, which brought the final sample size to 651 subjects. Participants presented for between 1 and 6 study visits, with $81 \%(n=529)$ of participants undergoing at least two study visits, and the mean length of follow-up was 4.68 years (range $0-16$ years).

Detailed questionnaires and physical examinations were administered at all centre-based study visits, which occurred every 3-5 years. Physical examinations included measurement of height and weight. Smoking history was obtained via an American Thoracic Society (ATS) questionnaire. Lung function, measured by $\mathrm{FVC}$ and $\mathrm{FEV}_{1}$, was measured using spirometric tests performed according to the ATS guidelines. ${ }^{2}$ At least three spirograms were taken, at least two of which were reproducible within 5\% for both FVC and $\mathrm{FEV}_{1}$. This study was approved by the institutional review boards of all participating institutions, and all participants provided their written informed consent.

\section{BC exposure assessment}

Exposures to BC prior to each study visit were calculated using validated spatiotemporal land use regression models. ${ }^{12}$ These models used BC data from 148 monitors distributed throughout the Greater Boston area. Exposures were estimated at participants' geocoded residential addresses up to the time of visit. Daily BC predictions were generated by the geographical models and combined into 1-year and 5-year moving averages of $\mathrm{BC}$ exposure prior to each study visit. ${ }^{2}$

\section{Genetic scores}

Participants' DNA was amplified using PCR, and genotyping was conducted using the Sequenom MassArray MALDI-TOF Mass Spectrometer (San Diego, California, USA), as described previously. ${ }^{10}$ This method yields high-quality data with high sensitivity. Eighteen polymorphisms associated with reactive oxygen species generation or antioxidative defences were evaluated for inclusion in the oxidative stress genetic score (CAT: rs2284367, rs1001179, rs2300181, rs480575; HMOX1: rs2071746, rs5995098, rs2071749, rs2071747, and short/long (<25 vs 25+ GT repeats); NQO1: rs1800566; GSTP1: rs1695, rs1799811; GC: rs2282679, rs1155563; GCLM: rs2301022, rs3170633; GSTM1: rs4147565 and a deletion polymorphism on chromosome 22). Of these, seven were selected using the least absolute shrinkage and selection operator (Lasso) based on their relation to 8-hydroxydeoxyguanosine levels (8-OhdG, a marker of oxidative DNA damage): CAT (rs1001179, rs480575), GC (rs2282679), GCLM (rs3170633), HMOX1 (rs2071746, rs5995098) and NQO1 (rs1800566). ${ }^{10}$ Scores representing participants' allelic profiles were constructed by summing these genetic variants, using the signs of the coefficients of the Lasso penalisation. ${ }^{10}$

\section{Statistical analyses}

Associations between increases in long-term BC exposure and lung function were calculated using separate linear mixed models for FVC and $\mathrm{FEV}_{1}{ }^{2}$ We examined effect modification of the BC-lung function association by oxidative stress using interaction terms between $\mathrm{BC}$ exposure and oxidative stress genetic score, which was dichotomised based on the median value (high vs low). ${ }^{10}$ The genetic score was dichotomised to enhance interpretability, as is standard when examining interactions with continuous variables. We chose to dichotomise at the median to maximise power for these analyses. Interaction $\mathrm{p}$ values of $<0.05$ were considered to be statistically significant. All statistical analyses were conducted using SAS V.9.4.

For each category of genetic score (high, low), we examined both the cross-sectional effect of $\mathrm{BC}$ exposure on $\mathrm{FVC}$ or $\mathrm{FEV}_{1}$ levels at baseline visit (ie, $\beta_{3}+\beta_{8}$, see below in equation (1)) and the effect of $\mathrm{BC}$ on the longitudinal rate of decline in lung function (ie, $\beta_{4}+\beta_{7}$, see below in equation (1)). We examined $0.5 \mu \mathrm{g} / \mathrm{m}^{3}$ as the BC exposure increment of interest to be consistent with a previous NAS report evaluating the main effects of long-term $\mathrm{BC}$ exposure on $\mathrm{FEV}_{1}$ and $\mathrm{FVC}^{2}$ and because this value corresponds closely with the overall mean exposure level in our study population. We also decided a priori to conduct sensitivity analyses with the genetic score dichotomised at the 75 th percentile. However, the 75 th percentile of the genetic score distribution was equal to the median genetic score in our study population, and hence the results were unchanged.

All models also controlled for the following covariates, identified via a thorough literature review: age, race (black or white), log-transformed height, weight (linear and quadratic terms), educational attainment $(<12,12,13-15$ or $\geq 15$ years $)$, smoking status (current, former and never), pack years of smoking and season ( $\sin ($ date) and $\cos ($ date)$)$. We evaluated whether inclusion of multiplicative interaction terms between genetic score and former and current smoking affected study results. The interaction terms were not statistically significant nor did their inclusion meaningfully alter any study findings. Hence, we omitted these interaction terms from the final models.

Our model for participant $i$ at time $t$ was:

$$
\begin{aligned}
& \log \left(\mathrm{FVC}_{\text {or FEV }}\right)_{\mathrm{it}}=\beta_{0}+\mu_{\mathrm{i}}+\beta_{1} \mathrm{Age}_{\text {baseline }, \mathrm{i}}+\beta_{2} \Delta \mathrm{Age}_{\text {baseline }, \mathrm{it}} \\
& +\beta_{3} \mathrm{BC}_{\mathrm{it}}+\beta_{4} \mathrm{BC}_{\mathrm{it}} * \Delta \mathrm{Age}_{\text {baseline,it }}+\beta_{5} \text { genetic score } \\
& +\beta_{6} \text { genetic score }^{*} \Delta \mathrm{Age}_{\text {baseline }, \mathrm{it}}+\beta_{7} \text { genetic score } * \\
& \Delta \mathrm{Age}_{\text {baseline,it }} * \mathrm{BC}+\beta_{8} \mathrm{BC}_{\mathrm{it}} * \text { genetic score }+\beta_{\mathrm{p}} \mathrm{X}_{\text {pit }}+\varepsilon_{\mathrm{it}}
\end{aligned}
$$

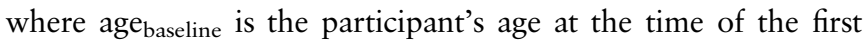
visit, $\mathrm{BC}$ is the average $\mathrm{BC}$ concentration at the home address of the participant for the preceding 1 or 5 years and $\Delta$ age is a time trend term measured in days since the baseline visit. Hence in this model $\beta_{3}$ measures the cross-sectional effect of $\mathrm{BC}$ at baseline, $\beta_{4}$ measures the effect of $\mathrm{BC}$ on the rate of decline in lung function, $\beta_{2}$ is the slope of lung function decline in the participant at the mean exposure (we have centred $B C$ ), $\beta_{6}$ is the effect of genetic score on the rate of decline in lung function at the mean exposure, $\beta_{7}$ is the effect of genetic score modifying the effect of $\mathrm{BC}$ on lung function decline, $\beta_{8}$ is the effect of genetic score modifying the effect of $\mathrm{BC}$ on lung function at baseline and $\mathrm{X}$ represents the $\mathrm{p}$ covariates controlled for in the model. 


\section{RESULTS}

We report descriptive statistics for NAS participants, stratified by oxidative stress genetic scores, in table 1. Distributions of participant characteristics were similar between the high and low oxidative stress score groups. For example, FVC and $\mathrm{FEV}_{1}$ were identical in both groups (3.4 and $2.5 \mathrm{~L}$, respectively). Among participants with genetic scores below the median, mean age was 69.3 years, $97.1 \%$ of participants were White, $68.8 \%$ were former smokers and 5.4\% were current smokers. Among those with higher genetic scores, mean age was 69.8 years, $98.1 \%$ of participants were White, $62.1 \%$ were former smokers and $6.9 \%$ were current smokers.

The distribution of $\mathrm{BC}$ exposure estimates stratified by genetic scores is presented in table 2. For example, among participants with a high oxidative stress genetic score, the 1-year moving average of BC at all study visits was $0.7 \mu \mathrm{g} / \mathrm{m}^{3}$ (5th-95th percentiles 0.29-1.29).

Both $\mathrm{FEV}_{1}$ and FVC declined with time in the NAS, as expected, with an average annual decline of $0.9 \%$ for $\mathrm{FEV}_{1}$ and $0.7 \%$ for FVC among those with complete information regarding long-term residential BC exposure and oxidative stress allelic profile. As previously reported, the rate of decline per year was higher in people with higher BC exposure. ${ }^{2}$ In the current analysis, a $0.5 \mu \mathrm{g} / \mathrm{m}^{3}$ increase in the 5 -year moving average of BC exposure was associated with a $0.7 \%$ additional annual decrease in both $\mathrm{FEV}_{1}(95 \% \mathrm{CI}-1.2$ to -0.3$)$ and $\mathrm{FVC}(95 \% \mathrm{CI}-1.1$ to 0.4$)$.

In subjects with a higher oxidative risk profile, BC was associated with a higher rate of decline in lung function than in

Table 1 Characteristics of elderly men $(n=651)$ participating in the Normative Aging Study (1995-2011), stratified by oxidative stress genetic scores*

\begin{tabular}{|c|c|c|}
\hline $\begin{array}{l}\text { Participant characteristics at } \\
\text { baseline visit }\end{array}$ & $\begin{array}{l}\text { Oxidative stress } \\
\text { genetic score below } \\
\text { the median }(n=276)\end{array}$ & $\begin{array}{l}\text { Oxidative stress } \\
\text { genetic score above } \\
\text { the median }(n=375)\end{array}$ \\
\hline Age in years (mean, SD) & $69.3(7.3)$ & $69.8(7.2)$ \\
\hline Height, m (mean, SD) & $1.7(0.1)$ & $1.7(0.1)$ \\
\hline Weight, kg (mean, SD) & $83.7(12.9)$ & $84.9(14.8)$ \\
\hline $\begin{array}{l}\text { Pack years of smoking } \dagger \\
\text { (median, IQR) }\end{array}$ & $27.0(36.0)$ & $22.0(36.5)$ \\
\hline FVC, L (mean, SD) & $3.4(0.7)$ & $3.4(0.7)$ \\
\hline $\mathrm{FEV}_{1}, \mathrm{~L}$ (mean, SD) & $2.5(0.6)$ & $2.5(0.6)$ \\
\hline \multicolumn{3}{|l|}{ Race $(\mathrm{n}, \%)$} \\
\hline Black & $8(2.9)$ & $7(1.9)$ \\
\hline White & $268(97.1)$ & $368(98.1)$ \\
\hline \multicolumn{3}{|l|}{ Educational attainment $(\mathrm{n}, \%)$} \\
\hline$<12$ years & $8(2.9)$ & $26(6.9)$ \\
\hline 12 years & 65 (23.6) & $88(23.5)$ \\
\hline $13-15$ years & $85(30.8)$ & $119(31.7)$ \\
\hline$\geq 15$ years & $118(42.8)$ & $142(37.9)$ \\
\hline \multicolumn{3}{|l|}{ Smoking status (n, \%) } \\
\hline Former & $190(68.8)$ & $233(62.1)$ \\
\hline Current & $15(5.4)$ & $26(6.9)$ \\
\hline Never & $71(25.7)$ & $116(30.9)$ \\
\hline \multicolumn{3}{|l|}{ Season $(n, \%)$} \\
\hline Spring (March-May) & $188(22.9)$ & $283(25.4)$ \\
\hline Summer (June-August) & $216(26.3)$ & $295(26.5)$ \\
\hline Fall (September-November) & $255(31.1)$ & $311(27.9)$ \\
\hline Winter (December-February) & $161(19.6)$ & $224(20.1)$ \\
\hline
\end{tabular}

Table 2 Black carbon exposure distribution in $\mu \mathrm{g} / \mathrm{m}^{3}$ among 651 men undergoing 1933 study visits in the Normative Aging Study (1995-2011), stratified by oxidative stress genetic scores*

\begin{tabular}{|c|c|c|c|c|}
\hline $\begin{array}{l}\text { Exposure } \\
\text { duration }\end{array}$ & $\begin{array}{l}\text { Number } \\
\text { of visits }\end{array}$ & $\begin{array}{l}\text { Mean } \\
\text { (SD) }\end{array}$ & $\begin{array}{l}\text { 5th-95th } \\
\text { percentiles }\end{array}$ & IQR \\
\hline \multicolumn{5}{|c|}{ Genetic scores below the median } \\
\hline 1 year & 820 & $0.6(0.3)$ & $0.28-1.23$ & $0.43-0.80$ \\
\hline 5 years & 524 & $0.6(0.3)$ & $0.28-1.13$ & $0.44-0.77$ \\
\hline \multicolumn{5}{|c|}{ Genetic scores above the median } \\
\hline 1 year & 1113 & $0.7(0.3)$ & $0.29-1.29$ & $0.46-0.84$ \\
\hline 5 years & 716 & $0.7(0.3)$ & $0.30-1.17$ & $0.46-0.82$ \\
\hline
\end{tabular}

subjects with a lower oxidative profile (table 3). For each $0.5 \mu \mathrm{g} /$ $\mathrm{m}^{3}$ increase in 1-year BC exposure, we report an additional rate of decline in FVC of $0.6 \%$ per year (95\% CI -1.0 to -0.3 ) among participants with low genetic scores and $1.1 \%$ per year (95\% CI -1.3 to -0.8 ) among those with high scores $(\mathrm{p}$-interaction $=0.04)$. For 5 -year BC exposure, we report an annual $0.1 \%$ increase in FVC (95\% CI -0.5 to 0.7 ) among those with low genetic scores and a $1.3 \%$ decrease $(95 \%$ CI -1.8 to -0.8 ) among those with high scores ( $\mathrm{p}$-interaction $=0.0003)$. We observed the same trend for $\mathrm{FEV}_{1}$ (table 3).

We found no consistent evidence that the effect of $\mathrm{BC}$ on baseline FVC and $\mathrm{FEV}_{1}$ was modified by oxidative stress allelic profiles (table 3). Compared with 1-year moving average exposure, 5-year moving averages of $\mathrm{BC}$ exposure were more strongly related to reduced baseline lung function among those with higher oxidative stress allelic risk profiles, but this contrast did not rise to statistical significance.

\section{DISCUSSION}

Our study found stronger associations with larger effect sizes between long-term exposure to BC, a traffic-related pollutant and increased rate of lung function decline among elderly men with higher oxidative stress allelic profiles, relative to those with a lower oxidative stress risk profile. Associations were strongest when evaluating 5-year rather than 1-year BC exposures. Our results build on previous findings from the NAS, which have shown clinically significant effects on lung function at particle levels below Environmental Protection Agency (EPA) standards. ${ }^{2}$

Most importantly, if confirmed, the present results suggest a susceptible subpopulation that should be taken into account when considering air-quality recommendations and risk assessments: elderly men with higher oxidative stress allelic risk profiles. The Clean Air Act requires that national air-quality standards protect the health of susceptible subpopulations. The standards currently in effect, however, may be insufficiently protective due, in part, to the sparsity of relevant studies of susceptibility, particularly with regard to genetic characteristics. ${ }^{13}$

Epidemiological and laboratory studies have consistently reported associations between overall particulate matter or traffic exposure and reduced lung function. ${ }^{1} 3414$ Such research among the elderly is sparse, although this sizeable subpopulation is more sensitive to pollutant effects due to age-related changes in lung structure and function, as well as global changes in physiological functioning. ${ }^{15}$ The relationship between lung function and exposure to $\mathrm{BC}$ specifically has also been sparsely investigated, but $\mathrm{BC}$ studies to date are consistent with the overall literature for particles and air pollution. For example, a study reported an association between BC exposure and 
Table 3 Effect modification of the association between a $0.5 \mu \mathrm{g} / \mathrm{m}^{3}$ increase in long-term black carbon exposure and lung function by oxidative stress gene scores, Normative Aging Study (1995-2011)*

\begin{tabular}{|c|c|c|c|c|c|}
\hline \multirow[b]{2}{*}{ Exposure window } & \multirow[b]{2}{*}{ Number of visits } & \multicolumn{2}{|c|}{ Cross-sectional effect } & \multicolumn{2}{|c|}{ Rate of lung function decline } \\
\hline & & $\boldsymbol{\beta}(\%)$ & $95 \% \mathrm{Cl}$ & $\boldsymbol{\beta}(\%)$ & $95 \% \mathrm{Cl}$ \\
\hline \multicolumn{6}{|l|}{ FVC } \\
\hline \multicolumn{6}{|l|}{1 year } \\
\hline Gene score $<$ median & 822 & -1.4 & -3.4 to 0.6 & -0.6 & -1.0 to -0.3 \\
\hline Gene score >median & 1113 & -0.2 & -1.8 to 1.3 & -1.1 & -1.3 to -0.8 \\
\hline p For interaction & & $p=0.36$ & & $p=0.04$ & \\
\hline \multicolumn{6}{|l|}{5 years } \\
\hline Gene score $<$ median & 526 & -3.4 & -6.8 to 0.1 & 0.1 & -0.5 to 0.7 \\
\hline Gene score $>$ median & 716 & -6.7 & -9.4 to -4.0 & -1.3 & -1.8 to -0.8 \\
\hline $\mathrm{p}$ For interaction & & $p=0.14$ & & $p=0.0003$ & \\
\hline \multicolumn{6}{|l|}{ FEV $_{1}$} \\
\hline \multicolumn{6}{|l|}{1 year } \\
\hline Gene score $<$ median & 822 & -2.7 & -4.9 to -0.4 & -0.2 & -0.6 to 0.1 \\
\hline Gene score $>$ median & 1113 & -0.1 & -1.8 to 1.7 & -0.6 & -0.9 to -0.4 \\
\hline $\mathrm{p}$ For interaction & & $p=0.07$ & & $\mathrm{p}=0.08$ & \\
\hline \multicolumn{6}{|l|}{5 years } \\
\hline Gen score <median & 526 & -5.8 & -9.7 to 1.6 & -0.1 & -0.8 to 0.6 \\
\hline Gene score $>$ median & 716 & -6.9 & -10.1 to -3.6 & -1.2 & -1.7 to -0.6 \\
\hline$p$ For interaction & & $p=0.67$ & & $\mathrm{p}=0.01$ & \\
\hline
\end{tabular}

*All analyses were adjusted for age, race (Black or White), log-transformed height, weight (linear and quadratic terms), educational attainment ( $<12,12,13-15$ or $\geq 15$ years), smoking status (current, former and never), pack years of smoking and season (sin(date) and cos(date)).

reduced lung function among urban women, ${ }^{16}$ and short-term, subchronic and long-term BC exposures have been linked to reduced lung function within the current study population. ${ }^{2} 17$

Oxidative stress is the most likely pathway mediating the association between particles, including BC, and lung function changes. Particulate air pollution is known to induce oxidative stress systemically in the lung, ${ }^{89}$ and oxidative stress is important in the aetiology of pulmonary dysfunction. ${ }^{7}$ Hence, our findings of a stronger association between $\mathrm{BC}$ exposure and lung function reduction among men with higher oxidative stress allelic profiles is consistent with the biological literature to date. To our knowledge, no previous epidemiological studies have evaluated interactions between $\mathrm{BC}$ and oxidative stress genes with respect to pulmonary function, and this interaction has been very sparsely investigated for other particulate pollutants as well. Our findings are consistent, however, with the few relevant epidemiological studies conducted to date, which report interactions between several oxidative stress polymorphisms, tobacco smoke and particulate air pollutants. ${ }^{18} 19$

Limitations of our study include potential exposure misclassification of BC estimates, which is standard to environmental epidemiological studies. Our use of spatiotemporal land use regression models to estimate exposures at individual residences reduces exposure misclassification relative to fixed monitoring studies. Also, any BC measurement error is likely to be of the Berkson type, which would increase SEs without biasing the estimated effect size. ${ }^{20}$

Although loss to follow-up is inevitable in long-term longitudinal cohort studies, it should be noted that participants returning for subsequent visits may have been healthier than those not returning, and this study, like all longitudinal cohorts, may therefore be limited by a dynamic selection bias.

Our study population comprises elderly men, the vast majority of whom are retired, which is both a limitation and a strength. Participants' residential exposures are likely to reflect the great majority of their total exposure burden, which is a strength in terms of exposure assessment, but results may not be generalisable to other (non-elderly, non-White) segments of the population. However, we are specifically interested in the effect of air pollution on this potentially susceptible subgroup. Future research should explore the observed interactions further, using a range of ambient pollutants, oxidative stress variants and within different segments of the general population.

As previously mentioned, we found consistent evidence of interactions between oxidative stress genotype and BC exposure with respect to the rate of lung function decline, but not baseline lung function levels. Results for the longest term BC exposures, however, did suggest reduced baseline lung function among men with higher allelic risk profiles, though these results did not rise to the level of statistical significance. Differences in findings for the cross-sectional and longitudinal analyses may be due to residual confounding. Although we controlled carefully for potential confounders, there is greater potential for residual confounding in cross-sectional rather than longitudinal analyses; when evaluating changes over time in a study with repeated visits, individual-level confounders that change slowly over time tend to subtract out of the model. Also, the number of observations is smaller for the cross-sectional analyses, which could increase the rate of false-negative findings. Regardless, effects on lung function decline over time are likely to be more important from a clinical and public health perspective than effects on lung function at a single visit.

Strengths of our study include access to a large, general population cohort study with extensive and repeated information regarding environmental exposures, potential confounders and effect modifiers, and meticulously measured lung function data from multiple study visits. While most air pollution studies examine short-term exposure windows, long-term air pollution exposure may be of most interest when assessing risk for chronic disease and dysfunction. Hence, an additional strength 
of our study was the reconstruction of participants' long-term BC exposures using a comprehensive geographical model. Our use of highly individualised residential measurements is another important strength, since traffic exposures can vary significantly within a small geographical area. Finally, our use of oxidative stress genetic scores, designed in relation to their association with a valid marker of oxidative stress (8-OHdG), allowed us to effectively examine the biological mechanisms potentially underlying the $\mathrm{BC}$-lung function association, while reducing the number of comparisons.

\section{CONCLUSIONS}

We report stronger associations between long-term $\mathrm{BC}$ exposure and rate of lung function decline among elderly men with relatively high oxidative stress allelic risk profiles compared with men with a lower risk profile. This observation is biologically plausible, given the pro-oxidant effects of traffic pollution and the key role of oxidative stress in the aetiology of pulmonary dysfunction. Lung function decline is linked to reduced quality of life and increased mortality risk among the elderly, ${ }^{21}$ who are a vulnerable yet understudied population in terms of sensitivity to pollutant exposure, even at levels below EPA air-quality standards. ${ }^{1422}$ Our results, if confirmed, should inform air-quality recommendations and risk assessments in light of a susceptible subgroup.

\section{Author affiliations \\ ${ }^{1}$ Exposure, Epidemiology, and Risk Program, Department of Environmental Health, Harvard School of Public Health, Boston, Massachusetts, USA \\ 2INSERM, IAB, Team of Environmental Epidemiology applied to Reproduction and Respiratory Health, Grenoble, France \\ ${ }^{3}$ University of Grenoble Alpes, IAB, Team of Environmental Epidemiology applied to Reproduction and Respiratory Health, Grenoble, France \\ ${ }^{4} \mathrm{CHU}$ de Grenoble, IAB, Team of Environmental Epidemiology applied to Reproduction and Respiratory Health, Grenoble, France \\ ${ }^{5}$ Department of Biostatistics, Harvard School of Public Health, Boston, \\ Massachusetts, USA \\ ${ }^{6}$ VA Normative Aging Study, Veterans Affairs Boston Healthcare System and the Department of Medicine, Boston University School of Medicine, Boston, Massachusetts, USA \\ ${ }^{7}$ Channing Laboratory, Brigham and Women's Hospital, Harvard Medical School, Boston, Massachusetts, USA}

Contributors IM: principal contributor and guarantor, involved in designing and conducting statistical analyses, interpretation of results, drafting the manuscript and the review and approval of the manuscript. JL: involved in study conception and interpretation of the data, drafting, review and approval of the manuscript. BAC: involved in creating the study's environmental exposure model (ie, data acquisition), and in the drafting, review and approval of the manuscript. DS: involved in data acquisition and interpretation, and drafting, review and approval of the manuscript. PV: involved in data acquisition and drafting, review and approval of the manuscript. JS: involved in study conception, data acquisition, analysis, and interpretation, drafting of the manuscript and review and approval of the manuscript.

Funding This work was supported by the US Environmental Protection Agency grants R832416 and RD 83479801, by the National Institute of Environmental Health Sciences grants ES015172-01 and ES000002, and by a VA Research Career Scientist award to David Sparrow. The VA Normative Aging Study is supported by the Cooperative Studies Program/Epidemiology Research and Information Center of the US Department of Veterans Affairs and is a component of the Massachusetts Veterans Epidemiology Research and Information Center, Boston, Massachusetts.

Competing interests None.
Ethics approval Veterans Administration Institutional Review Board and Harvard School of Public Health Institutional Review Board.

Provenance and peer review Not commissioned; externally peer reviewed.

\section{REFERENCES}

1 Gauderman WJ, Avol E, Gilliland F, et al. The effect of air pollution on lung development from 10 to 18 years of age. N Engl J Med 2004;351:1057-67.

2 Lepeule J, Litonjua AA, Coull B, et al. Long-term effects of Traffic Particles on lung function decline in the elderly. Am J Respir Crit Care Med 2014;190:542-8.

3 Mauad T, Rivero DH, de Oliveira RC, et al. Chronic exposure to ambient levels of urban particles affects mouse lung development. Am J Respir Crit Care Med 2008; 178:721-8.

4 Rice MB, Ljungman PL, Wilker EH, et al. Short-term exposure to air pollution and lung function in the Framingham Heart Study. Am J Respir Crit Care Med 2013; 188:1351-7.

5 Neas LM, Schwartz J. Pulmonary function levels as predictors of mortality in a national sample of us adults. Am J Epidemiol 1998;147:1011-8.

6 Janssen NA, Hoek G, Simic-Lawson M, et al. Black carbon as an additional indicator of the adverse health effects of airborne particles compared with pm10 and pm2.5. Environ Health Perspect 2011;119:1691-9.

7 Ochs-Balcom HM, Grant BJ, Muti P, et al. Antioxidants, oxidative stress, and pulmonary function in individuals diagnosed with asthma or COPD. Eur J Clin Nutr 2006:60:991-9.

8 Evelson P, Gonzalez-Flecha B. Time course and quantitative analysis of the adaptive responses to $85 \%$ oxygen in the rat lung and heart. Biochim Biophys Acta 2000;1523:209-16.

9 Neophytou AM, Hart JE, Cavallari JM, et al. Traffic-related exposures and biomarkers of systemic inflammation, endothelial activation and oxidative stress: a panel study in the US trucking industry. Environ Health 2013;12:105.

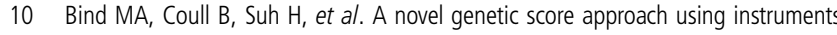
to investigate interactions between pathways and environment: application to air pollution. PloS ONE 2014;9:e96000.

11 Bell B, Rose C, Damon A. The Normative Aging Study: an interdisciplinary and longitudinal study of health and aging. Aging Hum Dev 1972;3:4-17.

12 Suglia SF, Gryparis A, Wright RO, et al. Association of black carbon with cognition among children in a prospective birth cohort study. Am J Epidemiol 2008;167:280-6.

13 Johnson PR, Graham JJ. Fine particulate matter national ambient air quality standards: public health impact on populations in the northeastern United States. Environ Health Perspect 2005;113:1140-7.

14 Rojas-Martinez R, Perez-Padilla R, Olaiz-Fernandez G, et al. Lung function growth in children with long-term exposure to air pollutants in Mexico City. Am J Respir Crit Care Med 2007;176:377-84.

15 Sharma G, Goodwin J. Effect of aging on respiratory system physiology and immunology. Clin Interv Aging 2006;1:253-60.

16 Suglia SF, Gryparis A, Schwartz J, et al. Association between traffic-related black carbon exposure and lung function among urban women. Environ Health Perspect 2008;116:1333-7.

17 Lepeule J, Bind MA, Baccarelli AA, et al. Epigenetic influences on associations between air pollutants and lung function in elderly men: The Normative Aging Study. Environ Health Perspect 2014;122:566-72.

18 Curjuric I, Imboden M, Nadif R, et al. Different genes interact with particulate matter and tobacco smoke exposure in affecting lung function decline in the general population. PloS ONE 2012;7:e40175.

19 Reddy P, Naidoo RN, Robins TG, et al. GSTM1 and GSTP1 gene variant and the effect of air pollutants on lung function measures in South African children. Am J Ind Med 2012;55:1078-86.

20 Zeger SL, Thomas D, Dominici F, et al. Exposure measurement error in time-series studies of air pollution: concepts and consequences. Environ Health Perspect 2000;108:419-26.

21 Mannino DM, Davis KJ. Lung function decline and outcomes in an elderly population. Thorax 2006;61:472-7.

22 Anderson JO, Thundiyil JG, Stolbach A. Clearing the air: a review of the effects of particulate matter air pollution on human health. J Med Toxicol 2012;8:166-75. 